\title{
All-order renormalization of propagator matrix for fermionic system with flavor mixing
}

\author{
Bernd A. Knieh $*$ \\ Kavli Institute for Theoretical Physics, University of California, Santa Barbara, CA 93106-4030, USA
}

(Dated: December 19, 2013)

\begin{abstract}
We consider a mixed system of Dirac fermions in a general parity-nonconserving theory and renormalize the propagator matrix to all orders in the pole scheme, in which the squares of the renormalized masses are identified with the complex pole positions and the wave-function renormalization (WFR) matrices are adjusted in compliance with the Lehmann-Symanzik-Zimmermann reduction formalism. We present closed analytic all-order expressions and their expansions through two loops for the renormalization constants in terms of the scalar, pseudoscalar, vector, and pseudovector parts of the unrenormalized self-energy matrix, which is computable from the one-particleirreducible Feynman diagrams of the flavor transitions. We identify residual degrees of freedom in the WFR matrices and propose an additional renormalization condition to exhaust them. We then explain how our results may be generalized to the case of unstable fermions, in which we encounter the phenomenon of WFR bifurcation. In the special case of a solitary unstable fermion, the all-order-renormalized propagator is presented in a particularly compact form.
\end{abstract}

PACS numbers: 11.10.Gh, 11.15.Bt, 12.15.Ff, 12.15.Lk

The experiments at the CERN Large Hadron Collider have radically changed the landscape of particle physics. In fact, a new weak neutral resonance, which very much looks like the missing link of the Standard Model (SM), has been discovered [1], while, despite concerted endeavors by armies of experimental and theoretical physicists, no signal of new physics beyond the SM has emerged so far. Within the present experimental precision, this new particle shares the spin, parity, and charge-conjugation quantum numbers $J^{P C}=0^{++}$ and the coupling strengths with the SM Higgs boson $H$, and its mass $(125.6 \pm 0.3) \mathrm{GeV}$ lies well inside the $M_{H}$ range predicted within the SM through global analyses of electroweak (EW) precision data, and it almost perfectly coincides with state-of-the-art determinations of the $M_{H}$ lower bound, $(129.6 \pm 1.5) \mathrm{GeV}$, from the requirement that the SM vacuum be stable way up to the scale of the Planck mass [2]. If the pole mass $m_{t}$ of the top quark, which, in want of a rigorous determination at the quantum level, is presently identified with a MonteCarlo parameter [3], were just lower by an amount of the order of its total decay width $\Gamma_{t}=(2.0 \pm 0.5) \mathrm{GeV}[3]$, then the agreement would be perfect, implying that EW symmetry breaking is likely to be determined by Planckscale physics. In a way, this would solve the longstanding hierarchy problem of the SM. The Nobel Prize in Physics 2013 has just been awarded jointly to Englert and Higgs for the theoretical discovery of the Higgs mechanism.

Obviously, nature is telling us that the SM is more robust and fundamental than commonly accepted in recent years. This provides a strong motivation for us to deepen and complete our understanding of the field-

\footnotetext{
*Permanent address: II. Institut für Theoretische Physik, Universität Hamburg, Luruper Chaussee 149, 22761 Hamburg, Germany.
}

theoretic foundations of the SM. After all, we are dealing here with a renormalizable quantum field theory [4]. The Nobel Prize in Physics 1999 was awarded to 't Hooft and Veltman for elucidating the quantum structure of EW interactions in physics. The on-shell renormalization of the SM was established to all orders of perturbation theory using the algebraic method [5]. However, all particles were assumed to be stable, neutrinos were taken to be massless, and quark flavor mixing was neglected. To eliminate these unrealistic assumptions, one needs to develop a pole scheme of mixing renormalization for unstable particles valid to all orders. Apart from being conceptually desirable, this is becoming of major phenomenological importance, even more so because mixing and instability of elementary particles concur in nature. This requires generalized concepts for flavor-changing propagators and vertices. In the SM with massless neutrinos, these are the propagator matrices of the up- and downtype quarks and their charged-current vertices, which involve the Cabibbo-Kobayashi-Maskawa (CKM) [] $]$ quark mixing matrix. This pattern carries over to the lepton sector if the neutrinos are massive Dirac fermions, and the analogue of the CKM matrix is the Pontecorvo-MakiNakagawa-Sakata 7] neutrino mixing matrix.

The renormalization of fermion mixing matrices was treated in Ref. [8] and the references cited therein. As for the renormalization of propagator matrices of mixed systems of fermions, the situation is as follows. In Ref. [9], an early treatment of finite renormalization effects both for quarks in hadronic bound states and leptons may be found. In Ref. [10], the ultraviolet (UV) renormalization of the fermion masses was considered, and the pole masses were shown to be gauge independent to all orders in the SM using Nielsen identities [11], both for stable and unstable fermions. In Ref. [12], the UV renormalization of the fermion fields was discussed for the case of stability, and the dressed propagator matrices were written in 
closed form, both for the unrenormalized and renormalized versions. Furthermore, it was explicitly proven that the WFR conditions proposed in Ref. 13] guarantee the unit-residue properties of the diagonal elements of the renormalized propagator matrix to all orders, in compliance with the Lehmann-Symanzik-Zimmermann (LSZ) reduction formalism [14]. The purpose of this Letter is to construct closed analytic expressions for the mass counterterms and WFR matrices without resorting to perturbation theory and to generalize the treatment to unstable fermions. Strictly speaking, unstable particles are not entitled to appear in asymptotic states of scattering amplitudes in quantum field theory. However, in numerous applications of significant phenomenological interest, the rigorous compliance with this tenet would immediately entail a proliferation of external legs and bring the evaluation of radiative corrections to a grinding halt, the more so as almost all the known elementary particles are unstable.

We consider a system of $N$ unstable Dirac fermions in the context of some general parity-nonconserving renormalizable quantum field theory with inter-generation mixing, such as the up-type or down-type quarks in the SM. We start from the bare theory and assume that the mass matrix has already been diagonalized. The bare masses $m_{i}^{0}$, where $i=1, \ldots, N$ is the generation index and the superscript 0 labels bare quantities, are real and non-negative to ensure the reality of the action and the absence of tachyons, respectively. For the sake of a compact notation, we group the bare quantum fields $\psi_{i}^{0}(x)$ into a column vector $\Psi^{0}(x)$. In momentum space, the unrenormalized propagator matrix is defined as $i P(\not p)=\int d^{4} x e^{i p \cdot x}\left\langle 0\left|T\left[\Psi^{0}(x) \bar{\Psi}^{0}(0)\right]\right| 0\right\rangle$, where $T$ is the time-ordered product, $\bar{\Psi}^{0}(x)=\left[\Psi^{0}(x)\right]^{\dagger} \gamma^{0}$, and a tensorial product both in the spinor and generation spaces is implied. Its inverse is built up by the one-particleirreducible Feynman diagrams contributing to the transitions $j \rightarrow i$ and takes the form

$$
P^{-1}(\not p)=\not p-M^{0}-\Sigma(\not p),
$$

where $M_{i j}^{0}=m_{i}^{0} \delta_{i j}$ and $\Sigma(\not p)$ is the unrenormalized selfenergy matrix. Here and in the following, repeated indices are not summed over unless indicated by a summation symbol. Lorentz covariance entails

$$
\Sigma(\not p)=\left[\not p B_{+}\left(p^{2}\right)+A_{+}\left(p^{2}\right)\right] a_{+}+(+\leftrightarrow-),
$$

where $a_{ \pm}=\left(1 \pm \gamma_{5}\right) / 2$ are the chiral projection operators and $\left[A_{ \pm}\left(p^{2}\right)\right]_{i j}$ and $\left[B_{ \pm}\left(p^{2}\right)\right]_{i j}$ are Lorentz invariants. The latter may be calculated from the bare Lagrangian order by order in perturbation theory. However, we refrain from resorting to perturbative expansions so as render our results valid to all orders. Defining

$$
S_{ \pm}\left(p^{2}\right)=\mathbb{1}-B_{ \pm}\left(p^{2}\right), \quad T_{ \pm}=M^{0}+A_{ \pm}\left(p^{2}\right),
$$

Eq. (11) becomes

$$
P^{-1}(\not p)=\left[\not p S_{+}\left(p^{2}\right)-T_{+}\left(p^{2}\right)\right] a_{+}+(+\leftrightarrow-) .
$$

Performing the Dyson resummation [15] is equivalent to inverting Eq. (41) and yields [12]

$$
\begin{aligned}
& P(\not p) \\
& =\left[\not p+D_{-}\left(p^{2}\right)\right] S_{-}^{-1}\left(p^{2}\right)\left[p^{2}-E_{-}\left(p^{2}\right)\right]^{-1} a_{+}+(+\leftrightarrow-) \\
& =a_{+}\left[p^{2}-F_{+}\left(p^{2}\right)\right]^{-1} S_{+}^{-1}\left(p^{2}\right)\left[\not p+C_{+}\left(p^{2}\right)\right]+(+\leftrightarrow-),
\end{aligned}
$$

with the short-hand notations

$$
\begin{aligned}
& C_{ \pm}\left(p^{2}\right)=T_{\mp}\left(p^{2}\right) S_{\mp}^{-1}\left(p^{2}\right), D_{ \pm}\left(p^{2}\right)=S_{\mp}^{-1}\left(p^{2}\right) T_{ \pm}\left(p^{2}\right), \\
& E_{ \pm}\left(p^{2}\right)=C_{ \pm}\left(p^{2}\right) C_{\mp}\left(p^{2}\right), F_{ \pm}\left(p^{2}\right)=D_{\mp}\left(p^{2}\right) D_{ \pm}\left(p^{2}\right),(6)
\end{aligned}
$$

where $S_{ \pm}^{-1}\left(p^{2}\right)=\sum_{n=0}^{\infty} B_{ \pm}^{n}\left(p^{2}\right)$ is a geometric series.

In the following, we shall exploit several times the following theorem for $n \times n$ matrices $A$ (for a proof, see e.g. Ref. [16]):

$$
A(\operatorname{adj} A)=(\operatorname{adj} A) A=(\operatorname{det} A) \mathbb{1},
$$

where $(\operatorname{adj} A)_{i j}=C_{j i}$ with $C_{i j}$ being the cofactor of $A_{i j}$, i.e. $(-1)^{i+j}$ times the determinant of the $(n-1) \times(n-1)$ matrix obtained by deleting the $i$-th row and the $j$ th column of $A$. If $\operatorname{det} A \neq 0$, then Eq. (7) implies that $A^{-1}=(\operatorname{adj} A) /(\operatorname{det} A)$. Since the four matrices $\left[p^{2}-E_{ \pm}\left(p^{2}\right)\right]$ and $\left[p^{2}-F_{ \pm}\left(p^{2}\right)\right]$ are related by similarity transformations, their determinants coincide. Owing to Eq. (7), the individual propagator parts in Eq. (5) thus all have their poles at the same (complex) positions $p^{2}=M_{i}^{2}$, which are the zeros of any of the secular equations [9, 10, 12]

$$
\operatorname{det}\left[M_{i}^{2}-E_{ \pm}\left(M_{i}^{2}\right)\right]=\operatorname{det}\left[M_{i}^{2}-F_{ \pm}\left(M_{i}^{2}\right)\right]=0 .
$$

Here, $M_{i}$ is the complex pole mass of fermion $i$, which is related to the real pole mass $m_{i}$ and total decay width $\Gamma_{i}$ as [17, 18]

$$
M_{i}=m_{i}-i \frac{\Gamma_{i}}{2} .
$$

In the pole renormalization scheme, $M_{i}$ serve as the renormalized masses, i.e. the mass counterterms $\delta M_{i}$ are fixed by

$$
m_{i}^{0}=M_{i}+\delta M_{i}
$$

We now turn to the WFR. We first assume that all the fermions are stable, with $\Gamma_{i}=0$, i.e. their mass shells $p^{2}=m_{i}^{2}$ lie below the thresholds of $\left[A_{ \pm}\left(p^{2}\right)\right]_{i j}$ and $\left[B_{ \pm}\left(p^{2}\right)\right]_{i j}$, where the absorptive parts of the latter vanish. The WFR is implemented by writing

$$
\Psi^{0}(x)=Z^{1 / 2} \Psi(x),
$$

where $\Psi(x)$ is the renormalized field multiplet and

$$
Z^{1 / 2}=Z_{+}^{1 / 2} a_{+}+Z_{-}^{1 / 2} a_{-}
$$

is the WFR matrix. Using Eq. (11), we may express the renormalized propagator matrix $i \hat{P}(\not p)=$ 
$\int d^{4} x e^{i p \cdot x}\langle 0|T[\Psi(x) \bar{\Psi}(0)]| 0\rangle$ in terms of the unrenormalized one as

$$
\hat{P}(\not p)=Z^{-1 / 2} P(\not p) \bar{Z}^{-1 / 2}
$$

where

$$
\bar{Z}^{1 / 2}=\gamma^{0} Z^{\dagger 1 / 2} \gamma^{0}=a_{-} Z_{+}^{\dagger 1 / 2}+a_{+} Z_{-}^{\dagger 1 / 2} .
$$

We may absorb the WFR matrices in Eq. (13) by introducing renormalized counterparts of $S_{ \pm}$and $T_{ \pm}$in Eq. (3),

$$
\hat{S}_{ \pm}\left(p^{2}\right)=Z_{ \pm}^{\dagger 1 / 2} S_{ \pm}\left(p^{2}\right) Z_{ \pm}^{1 / 2}, \quad \hat{T}_{ \pm}\left(p^{2}\right)=Z_{\mp}^{\dagger 1 / 2} T_{ \pm}\left(p^{2}\right) Z_{ \pm}^{1 / 2} .
$$

Feeding Eq. (15) into Eq. (6), we are thus led to define

$$
\begin{aligned}
& \hat{C}_{ \pm}\left(p^{2}\right)=\hat{T}_{\mp}\left(p^{2}\right) \hat{S}_{\mp}^{-1}\left(p^{2}\right)=Z_{ \pm}^{\dagger 1 / 2} C_{ \pm}\left(p^{2}\right) Z_{\mp}^{\dagger-1 / 2} \\
& \hat{D}_{ \pm}\left(p^{2}\right)=\hat{S}_{\mp}^{-1}\left(p^{2}\right) \hat{T}_{ \pm}\left(p^{2}\right)=Z_{\mp}^{-1 / 2} D_{ \pm}\left(p^{2}\right) Z_{ \pm}^{1 / 2} \\
& \hat{E}_{ \pm}\left(p^{2}\right)=\hat{C}_{ \pm}\left(p^{2}\right) \hat{C}_{\mp}\left(p^{2}\right)=Z_{ \pm}^{\dagger 1 / 2} E_{ \pm}\left(p^{2}\right) Z_{ \pm}^{\dagger-1 / 2} \\
& \hat{F}_{ \pm}\left(p^{2}\right)=\hat{D}_{\mp}\left(p^{2}\right) \hat{D}_{ \pm}\left(p^{2}\right)=Z_{ \pm}^{-1 / 2} F_{ \pm}\left(p^{2}\right) Z_{ \pm}^{1 / 2}
\end{aligned}
$$

The renormalized counterparts of Eqs. (4) and (5) then simply emerge by placing carets. From Eq. (16), we learn that the matrices $\hat{E}_{ \pm}\left(p^{2}\right)$ and $E_{ \pm}\left(p^{2}\right)\left(\hat{F}_{ \pm}\left(p^{2}\right)\right.$ and $\left.F_{ \pm}\left(p^{2}\right)\right)$ are similar, which implies that their determinants coincide. Hence, the pole positions $M_{i}^{2}$ fixed by Eq. (8) are not affected by the WFR [10].

In accordance with the LSZ reduction formalism [14], we determine $Z^{1 / 2}$ by requiring that, if the mass shell of a fermion is reached, the respective diagonal element of the renormalized propagator matrix resonates with unit residue, while the other elements stay finite, i.e.

$$
[\hat{P}(\not p)]_{i j}=\frac{\delta_{i n} \delta_{n j}}{\not p-M_{n}}+\mathcal{O}(1)
$$

in the limit $p^{2} \rightarrow M_{n}^{2}$. For $\Gamma_{i}=0$, this may be achieved by imposing the on-shell WFR conditions [13],

$$
\begin{aligned}
{\left[\hat{P}^{-1}(\not p)\right]_{i j} u\left(\vec{p}, M_{j}\right) } & =0, \\
\bar{u}\left(\vec{p}, M_{i}\right)\left[\hat{P}^{-1}(\not p)\right]_{i j} & =0, \\
\left\{\frac{1}{\not p-M_{i}}\left[\hat{P}^{-1}(\not p)\right]_{i i}\right\} u\left(\vec{p}, M_{i}\right) & =u\left(\vec{p}, M_{i}\right), \\
\bar{u}\left(\vec{p}, M_{i}\right)\left\{\left[\hat{P}^{-1}(\not p)\right]_{i i} \frac{1}{\not p-M_{i}}\right\} & =\bar{u}\left(\vec{p}, M_{i}\right),
\end{aligned}
$$

for all $i, j=1, \ldots, N$, where $u\left(\vec{p}, M_{i}\right)$ is a four-component spinor satisfying the Dirac equation $\left(\not p-M_{i}\right) u\left(\vec{p}, M_{i}\right)=0$ and $\bar{u}\left(\vec{p}, M_{i}\right)=\left[u\left(\vec{p}, M_{i}\right)\right]^{\dagger} \gamma^{0}$. For $\Gamma_{i}=0$, an explicit proof that Eqs. (18)-(21) entail Eq. (17) may be found in Sec. III of Ref. 12]. Equations (18)-(20) imply that

$$
\begin{aligned}
0= & {\left[\hat{S}_{\mp}\left(M_{j}^{2}\right)\right]_{i j} M_{j}-\left[\hat{T}_{ \pm}\left(M_{j}^{2}\right)\right]_{i j}, } \\
0= & M_{i}\left[\hat{S}_{ \pm}\left(M_{i}^{2}\right)\right]_{i j}-\left[\hat{T}_{ \pm}\left(M_{i}^{2}\right)\right]_{i j}, \\
1= & {\left[\hat{S}_{+}\left(M_{i}^{2}\right)\right]_{i i}+M_{i}^{2}\left\{\left[\hat{S}_{+}^{\prime}\left(M_{i}^{2}\right)\right]_{i i}+\left[\hat{S}_{-}^{\prime}\left(M_{i}^{2}\right)\right]_{i i}\right\} } \\
& -M_{i}\left\{\left[\hat{T}_{+}^{\prime}\left(M_{i}^{2}\right)\right]_{i i}+\left[\hat{T}_{-}^{\prime}\left(M_{i}^{2}\right)\right]_{i i}\right\},
\end{aligned}
$$

respectively, while Eq. (21) is redundant. Equation (20) also implies that $\left[\hat{S}_{+}\left(M_{i}^{2}\right)\right]_{i i}=\left[\hat{S}_{-}\left(M_{i}^{2}\right)\right]_{i i}$, which, however, already follows from Eqs. (22) and (23) for $i=j$.

We now solve Eqs. (22)-(24) exactly for $M_{i}, Z^{1 / 2}$, and $Z^{\dagger 1 / 2}$, without recourse to perturbation theory. Multiplying Eq. (22) by $\left[\hat{S}_{\mp}^{-1}\left(M_{j}^{2}\right)\right]_{k i}$ from the left, summing over $i$, iterating the outcome, and proceeding analogously with Eq. (23), we obtain the following eigenvalue equations:

$$
\begin{aligned}
{\left[F_{ \pm}\left(M_{j}^{2}\right) Z_{ \pm}^{1 / 2}\right]_{i j} } & =\left(Z_{ \pm}^{1 / 2}\right)_{i j} M_{j}^{2} \\
{\left[Z_{ \pm}^{\dagger 1 / 2} E_{ \pm}\left(M_{i}^{2}\right)\right]_{i j} } & =M_{i}^{2}\left(Z_{ \pm}^{\dagger 1 / 2}\right)_{i j}
\end{aligned}
$$

With the aid of Eqs. (7) and (8), we find solutions of the form

$$
\left(Z_{ \pm}^{1 / 2}\right)_{i j}=M_{i j}^{ \pm} \lambda_{j}^{ \pm}, \quad\left(Z_{ \pm}^{\dagger 1 / 2}\right)_{i j}=\bar{\lambda}_{i}^{ \pm} \bar{M}_{i j}^{ \pm}
$$

where $\lambda_{i}^{ \pm}$and $\bar{\lambda}_{i}^{ \pm}$are constants yet to be determined and

$$
\begin{aligned}
& M_{i j}^{ \pm}=\left\{\operatorname{adj}\left[M_{j}^{2}-F_{ \pm}\left(M_{j}^{2}\right)\right]\right\}_{i j}, \\
& \bar{M}_{i j}^{ \pm}=\left\{\operatorname{adj}\left[M_{i}^{2}-E_{ \pm}\left(M_{i}^{2}\right)\right]\right\}_{i j} .
\end{aligned}
$$

Substituting Eq. (26) into Eqs. (22) and (23) with $i=j$ and Eq. (24), we have

$$
\begin{aligned}
& M_{i} \bar{\lambda}_{i}^{+} s_{i}^{+} \lambda_{i}^{+}=M_{i} \bar{\lambda}_{i}^{-} s_{i}^{-} \lambda_{i}^{-}=\bar{\lambda}_{i}^{-} t_{i}^{+} \lambda_{i}^{+}=\bar{\lambda}_{i}^{+} t_{i}^{-} \lambda_{i}^{-}, \\
& \bar{\lambda}_{i}^{+} s_{i}^{+} \lambda_{i}^{+}+M_{i}^{2}\left(\bar{\lambda}_{i}^{+} s_{i}^{+\prime} \lambda_{i}^{+}+\bar{\lambda}_{i}^{-} s_{i}^{-\prime} \lambda_{i}^{-}\right) \\
& -M_{i}\left(\bar{\lambda}_{i}^{-} t_{i}^{+\prime} \lambda_{i}^{+}+\bar{\lambda}_{i}^{+} t_{i}^{-\prime} \lambda_{i}^{-}\right)=1,
\end{aligned}
$$

where

$s_{i}^{ \pm}\left(p^{2}\right)=\left[\bar{M}^{ \pm} S_{ \pm}\left(p^{2}\right) M^{ \pm}\right]_{i i}, t_{i}^{ \pm}\left(p^{2}\right)=\left[\bar{M}^{\mp} T_{ \pm}\left(p^{2}\right) M^{ \pm}\right]_{i i}$,

and $p^{2}=M_{i}^{2}$ is implied whenever the arguments are omitted. From Eq. (28), we obtain

$$
M_{i}^{2}=f_{i}\left(M_{i}^{2}\right)
$$

where

$$
f_{i}\left(p^{2}\right)=\frac{t_{i}^{+}\left(p^{2}\right) t_{i}^{-}\left(p^{2}\right)}{s_{i}^{+}\left(p^{2}\right) s_{i}^{-}\left(p^{2}\right)} .
$$

Factoring out $\bar{\lambda}_{i}^{+} s_{i}^{+} \lambda_{i}^{+}$in Eq. (29) and using Eqs. (28), (31), and (32), we find

$$
\bar{\lambda}_{i}^{+} s_{i}^{+} \lambda_{i}^{+}\left[1-f_{i}^{\prime}\left(M_{i}^{2}\right)\right]=1 .
$$

Using Eq. (26) for $i=j$ and Eq. (28), we arrive at

$$
\begin{aligned}
\left(Z_{ \pm}^{\dagger 1 / 2}\right)_{i i}\left(Z_{ \pm}^{1 / 2}\right)_{i i} & =\frac{\bar{M}_{i i}^{ \pm} M_{i i}^{ \pm}}{s_{i}^{ \pm}\left[1-f_{i}^{\prime}\left(M_{i}^{2}\right)\right]} \\
\left(Z_{\mp}^{\dagger 1 / 2}\right)_{i i}\left(Z_{ \pm}^{1 / 2}\right)_{i i} & =\frac{M_{i} \bar{M}_{i i}^{\mp} M_{i i}^{ \pm}}{t_{i}^{ \pm}\left[1-f_{i}^{\prime}\left(M_{i}^{2}\right)\right]} .
\end{aligned}
$$

The nondiagonal entities are then fixed by Eq. (26) to be

$$
\left(Z_{ \pm}^{1 / 2}\right)_{i j}=\frac{M_{i j}^{ \pm}}{M_{j j}^{ \pm}}\left(Z_{ \pm}^{1 / 2}\right)_{j j},\left(Z_{ \pm}^{\dagger 1 / 2}\right)_{i j}=\left(Z_{ \pm}^{\dagger 1 / 2}\right)_{i i} \frac{\bar{M}_{i j}^{ \pm}}{\bar{M}_{i i}^{ \pm}}
$$


Owing to our assumption $\Gamma_{i}=0$, the bare propagator matrix satisfies the pseudo-Hermiticity condition $\gamma^{0} P^{\dagger}(\not p) \gamma^{0}=P(\not p)$ [13], which implies that $A_{ \pm}^{\dagger}\left(p^{2}\right)=$ $A_{\mp}\left(p^{2}\right)$ and $B_{ \pm}^{\dagger}\left(p^{2}\right)=B_{ \pm}\left(p^{2}\right)[19,[20]$. Hence, we have $F_{ \pm}^{\dagger}\left(p^{2}\right)=E_{ \pm}\left(p^{2}\right),\left(M^{ \pm}\right)^{\dagger}=\bar{M}^{ \pm},\left[s_{i}^{ \pm}\left(p^{2}\right)\right]^{*}=s_{i}^{ \pm}\left(p^{2}\right)$, $\left[t_{i}^{ \pm}\left(p^{2}\right)\right]^{*}=t_{i}^{\mp}\left(p^{2}\right)$, and $\left[f_{i}\left(p^{2}\right)\right]^{*}=f_{i}\left(p^{2}\right)$. Consequently, the r.h.s. of Eq. (34) is real, as required by the l.h.s. being $\left|\left(Z_{ \pm}^{1 / 2}\right)_{i i}\right|^{2}$, and complex conjugation of Eq. (35) entails a flip of the alternating-sign labels on both sides. Furthermore, Eqs. (31) and (34)-36) are consistent with each other. For each value of $i$, Eqs. (34) and (35) provide four real equations for the four real unknowns $\operatorname{Re}\left(Z_{ \pm}^{1 / 2}\right)_{i i}$ and $\operatorname{Im}\left(Z_{ \pm}^{1 / 2}\right)_{i i}$. However, one of these equations is redundant due to Eq. (31). We may exhaust this residual freedom by choosing e.g. $\left(Z_{+}^{\dagger 1 / 2}\right)_{i i}=\left(Z_{+}^{1 / 2}\right)_{i i}$, as in Ref. 19]. This freedom does not affect Eq. (17). In fact, Eqs. (31) and (34) -(36) are valid to all orders. At one loop, they agree with Eqs. (3.13) and (3.15)-(3.17) in Ref. [19] and with Eqs. (3.3), (3.4), (4.3), and (4.4) in Ref. 20]. At two loops, Eq. (31) coincides with Eq. (23) in Ref. 12], which was found there by directly solving Eq. (8).

We now allow for some or all of the fermions to be unstable, releasing $M_{i}$ to complex values. This immediately leads to contradictions because the r.h.s. of Eq. (34) is no longer real and that of Eq. (35) no longer flips the alternating-sign labels upon complex conjugation, while the 1.h.s.'s still possess these properties. This problem may be cured by allowing the WFR matrices of the in and out states to bifurcate when $\Gamma_{i}$ increase to assume their physical values, as was already noticed in the pioneering one-loop analysis of Ref. [20]. This amounts to abandoning the first equality in Eq. (14) and replacing everywhere $Z_{ \pm}^{\dagger}$ by $\bar{Z}_{ \pm}$, say. Since the above manipulations of Eq. (13) actually never rely on the relationship between Eqs. (12) and (14), the derivation of Eqs. (31) and (34)(36) carries over without further ado, and so does the proof [12] that Eqs. (18)-(21) guarantee Eq. (17). For each value of $i$, Eqs. (34) and (35) now provide four complex equations for the four complex unknowns $\left(Z_{ \pm}^{1 / 2}\right)_{i i}$ and $\left(\bar{Z}_{ \pm}^{1 / 2}\right)_{i i}$. However, one of these equations is redundant, and we may express any three of the unknowns in terms of the fourth one. We may exploit this liberty e.g. by identifying $\left(\bar{Z}_{+}^{1 / 2}\right)_{i i}=\left(Z_{+}^{1 / 2}\right)_{i i}$. Again, this does not affect Eq. (17). From Eqs. (10) and (31), we obtain the all-order mass counterterms as

$$
\delta M_{i}=m_{i}^{0}-\sqrt{f_{i}\left(M_{i}^{2}\right)}
$$

Using also Eq. (9), we have

$$
\begin{aligned}
m_{i} & =\operatorname{Re} \sqrt{f_{i}\left(M_{i}^{2}\right)}=m_{i}^{0}-\operatorname{Re} \delta M_{i}, \\
-\frac{\Gamma_{i}}{2} & =\operatorname{Im} \sqrt{f_{i}\left(M_{i}^{2}\right)}=-\operatorname{Im} \delta M_{i} .
\end{aligned}
$$

Expanding the building blocks of Eqs. (31) and (34)-
(36) through $\mathcal{O}\left(\alpha^{2}\right)$, we find

$$
\begin{aligned}
f_{i}\left(p^{2}\right)= & \frac{\left[T_{+}\left(p^{2}\right)\right]_{i i}\left[T_{-}\left(p^{2}\right)\right]_{i i}}{\left[S_{+}\left(p^{2}\right)\right]_{i i}\left[S_{-}\left(p^{2}\right)\right]_{i i}}+m_{i}^{0}\left[\tau_{i}^{+}\left(p^{2}\right)+\tau_{i}^{-}\left(p^{2}\right)\right] \\
& -\left(m_{i}^{0}\right)^{2}\left[\sigma_{i}^{+}\left(p^{2}\right)+\sigma_{i}^{-}\left(p^{2}\right)\right]+\mathcal{O}\left(\alpha^{3}\right), \\
\frac{s_{i}^{ \pm}\left(p^{2}\right)}{\bar{M}_{i i}^{ \pm} M_{i i}^{ \pm}}= & {\left[S_{ \pm}\left(p^{2}\right)\right]_{i i}+\sigma_{i}^{ \pm}\left(p^{2}\right)+\mathcal{O}\left(\alpha^{3}\right), } \\
\frac{t_{i}^{ \pm}\left(p^{2}\right)}{\bar{M}_{i i}^{\mp} M_{i i}^{ \pm}}= & {\left[T_{ \pm}\left(p^{2}\right)\right]_{i i}+\tau_{i}^{ \pm}\left(p^{2}\right)+\mathcal{O}\left(\alpha^{3}\right), } \\
\frac{M_{j i}^{ \pm}}{M_{i i}^{ \pm}}= & f_{i j i}^{ \pm}\left(1+f_{i j j}^{ \pm}\right)+\sum_{i \neq k \neq j} f_{i j k}^{ \pm} f_{i k i}^{ \pm}+\mathcal{O}\left(\alpha^{3}\right), \\
\frac{\bar{M}_{i j}^{ \pm}}{\bar{M}_{i i}^{ \pm}}= & e_{i i j}^{ \pm}\left(1+e_{i j j}^{ \pm}\right)+\sum_{i \neq k \neq j} e_{i i k}^{ \pm} f_{i k j}^{ \pm}+\mathcal{O}\left(\alpha^{3}\right), \quad(40)
\end{aligned}
$$

for $j \neq i$, where

$$
\begin{aligned}
\sigma_{i}^{ \pm}\left(p^{2}\right)= & \sum_{j \neq i}\left\{e_{i i j}^{ \pm} f_{i j i}^{ \pm}-e_{i i j}^{ \pm}\left[B_{ \pm}\left(p^{2}\right)\right]_{j i}-\left[B_{ \pm}\left(p^{2}\right)\right]_{i j} f_{i j i}^{ \pm}\right\} \\
\tau_{i}^{ \pm}\left(p^{2}\right)= & \sum_{j \neq i}\left\{e_{i i j}^{\mp} m_{j}^{0} f_{i j i}^{ \pm}+e_{i i j}^{\mp}\left[A_{ \pm}\left(p^{2}\right)\right]_{j i}\right. \\
& \left.+\left[A_{ \pm}\left(p^{2}\right)\right]_{i j} f_{i j i}^{ \pm}\right\}, \\
f_{i j k}^{ \pm}= & \frac{\left.F_{ \pm}\left(M_{i}^{2}\right)\right]_{j k}-M_{j}^{2} \delta_{j k}}{M_{i}^{2}-M_{j}^{2}} \quad(j \neq i), \\
e_{i j k}^{ \pm}= & \frac{\left.E_{ \pm}\left(M_{i}^{2}\right)\right]_{j k}-\delta_{j k} M_{k}^{2}}{M_{i}^{2}-M_{k}^{2}} \quad(k \neq i) .
\end{aligned}
$$

We now consider the special case of a single unstable fermion, in which Eqs. (31) and (34)-(36) collapse and uniquely determine the renormalized propagator to be

$$
\begin{aligned}
\hat{P}(\not p)= & {\left[\not p+M \frac{S_{+}\left(M^{2}\right)}{S_{+}\left(p^{2}\right)} \frac{T_{-}\left(p^{2}\right)}{T_{-}\left(M^{2}\right)}\right] \frac{S_{-}\left(M^{2}\right)}{S_{-}\left(p^{2}\right)} } \\
& \times \frac{1-f^{\prime}\left(M^{2}\right)}{p^{2}-f\left(p^{2}\right)} a_{+}+(+\leftrightarrow-),
\end{aligned}
$$

where $f\left(p^{2}\right)=T_{+}\left(p^{2}\right) T_{-}\left(p^{2}\right) /\left[S_{+}\left(p^{2}\right) S_{-}\left(p^{2}\right)\right]$ and $M^{2}=$ $f\left(M^{2}\right)$. Evidently, Eq. (42) has unit residue at the physical pole $\not p=M$. We note that Eq. (42) slightly differs from Eq. (36) in Ref. [18], where a renormalization scheme without WFR bifurcation was employed.

In summary, we renormalized the propagator matrix of a mixed system of Dirac fermions in a general paritynonconserving quantum field theory adopting the pole scheme, in which the pole masses $M_{i}$ serve as the renormalized masses and the WFR matrices $Z_{ \pm}^{1 / 2}$ and $Z_{ \pm}^{\dagger^{1 / 2}}$ are adjusted in compliance with the LSZ reduction formalism [14. We derived closed analytic expressions for the renormalization constants in terms of the scalar, pseudoscalar, vector, and pseudovector parts of the unrenormalized self-energy matrix. These are valid to all orders and reproduce the results available in the literature, for $M_{i}$ at one [19, 20] and two loops [12] and for $Z_{ \pm}^{1 / 2}$ and $Z_{ \pm}^{\dagger 1 / 2}$ at one loop [19, 20]. We identified residual freedom 
in the determination of $Z_{ \pm}^{1 / 2}$ and $Z_{ \pm}^{\dagger 1 / 2}$ and proposed an additional renormalization condition to exhaust it. We then explained how our results carry over from stable fermions to unstable ones. In the latter case, we encountered WFR bifurcation, i.e. the departure from the first equality in Eq. (14), confirming the findings of Ref. [20] at one loop.

Apart from being conceptually interesting in their own right, our results have a number of important phenomenological applications, of which we mention but three below. First, in the perturbative treatment of a specific particle scattering or decay process involving unstable fermions, such as top-quark production and decay, Eqs. (31) and (34)-(36) may be readily employed, after expansion through the considered order and truncation of terms beyond that order. Second, the total decay width $\Gamma_{i}$, e.g. that of the top quark, may be conveniently evaluated through any order from $\left[A_{ \pm}\left(p^{2}\right)\right]_{i j}$ and $\left[B_{ \pm}\left(p^{2}\right)\right]_{i j}$ by solving Eq. (39) iteratively. Third, Eqs. (10) and (37) may be used to switch from the pole scheme adopted here to any other scheme of mass renormalization, as long as the method of regularization is maintained, exploiting the scheme independence of $m_{i}^{0}$. In this way, the $\overline{\mathrm{MS}}$ [21] definition of mass may be naturally extended from QCD to the EW sector, as

$$
\bar{m}_{i}=m_{i}+\left(\operatorname{Re} \delta M_{i}\right) \overline{\mathrm{MS}}
$$

where $\left(\operatorname{Re} \delta M_{i}\right)_{\overline{\mathrm{MS}}}$ is the UV-finite remainder of $\operatorname{Re} \delta M_{i}$ after $\overline{\mathrm{MS}}$ subtraction of the poles in $\varepsilon=2-d / 2$ at renormalization scale $\mu$, where $d$ is the dimensionality of space time in dimensional regularization 22]. In spontaneously broken gauge theories, such as the SM, it is necessary to include the tadpole contributions in $\left(\operatorname{Re} \delta M_{i}\right)_{\overline{\mathrm{MS}}}$ in order for $\bar{m}_{i}$ to be gauge independent [23]. In the case of the top quark, the accumulated QCD contribution to $\left(\operatorname{Re} \delta M_{t}\right) \overline{\mathrm{MS}}$ from orders $\mathcal{O}\left(\alpha_{s}^{n}\right)$ with $n=1,2,3$, which renders $\bar{m}_{t}$ at $\mu=m_{t}$ approximately $10 \mathrm{GeV}$ smaller than $m_{t}$, happens to be almost perfectly compensated by the EW contribution from orders $\mathcal{O}\left(\alpha \alpha_{s}^{n}\right)$ with $n=0,1$ for $M_{H} \approx 126 \mathrm{GeV}$ 24].

We are indebted to Alberto Sirlin for numerous beneficial discussions. This research was supported in part by DFG Grant No. SFB 676 and by NSF Grant No. PHY1125915.
[1] G. Aad et al. (ATLAS Collaboration), Phys. Lett. B 716, 1 (2012); S. Chatrchyan et al. (CMS Collaboration), ibid. 716, 30 (2012).

[2] F. Bezrukov, M. Yu. Kalmykov, B. A. Kniehl, and M. Shaposhnikov, J. High Energy Phys. HEP 10 (2012) 140; G. Degrassi, S. Di Vita, J. Elias-Miro, J. R. Espinosa, G. F. Giudice, G. Isidori, and A. Strumia, ibid. 08 (2012) 098; D. Buttazzo, G. Degrassi, P. P. Giardino, G. F. Giudice, F. Sala, A. Salvio, and A. Strumia, arXiv:1307.3536 [hep-ph].

[3] J. Beringer et al. (Particle Data Group), Phys. Rev. D 86, 010001 (2012).

[4] G. 't Hooft, Nucl. Phys. B33, 173 (1971); B35, 167 (1971); J. C. Taylor, ibid. B33 (1971) 436; B. W. Lee, Phys. Rev. D 5, 823 (1972); D. A. Ross and J. C. Taylor, Nucl. Phys. B51, 125 (1973); B58, 643(E) (1973).

[5] P. A. Grassi, Nucl. Phys. B462, 524 (1996); E. Kraus, Annals Phys. 262, 155 (1998).

[6] N. Cabibbo, Phys. Rev. Lett. 10, 531 (1963); M. Kobayashi and T. Maskawa, Prog. Theor. Phys. 49, 652 (1973).

[7] B. Pontecorvo, Zh. Eksp. Teor. Fiz. 33, 549 (1957); 34, 24 (1957) [Sov. Phys. JETP 6, 429 (1957); 7, 172 (1958)]; Z. Maki, M. Nakagawa, and S. Sakata, Prog. Theor. Phys. 28, 870 (1962).

[8] B. A. Kniehl and A. Sirlin, Phys. Rev. Lett. 97, 221801 (2006); Phys. Rev. D 74, 116003 (2006); Phys. Lett. B 673, 208 (2009).

[9] J. F. Donoghue, Phys. Rev. D 19, 2772 (1979).

[10] P. Gambino and P. A. Grassi, Phys. Rev. D 62, 076002 (2000).
[11] N. K. Nielsen, Nucl. Phys. B101, 173 (1975).

[12] B. A. Kniehl and A. Sirlin, Phys. Rev. D 85, 036007 (2012).

[13] K. I. Aoki, Z. Hioki, M. Konuma, R. Kawabe, and T. Muta, Prog. Theor. Phys. Suppl. 73, 1 (1982).

[14] H. Lehmann, K. Symanzik, and W. Zimmermann, Nuovo Cim. 1, 205 (1955); 6, 319 (1957).

[15] F. J. Dyson, Phys. Rev. 75, 1736 (1949).

[16] G. Birkhoff and S. Mac Lane, A Survey of Modern Algebra, Macmillan, New York, 1941; S. Perlis, Theory of Matrices, Addison-Wesley, Cambridge, 1952.

[17] M. C. Smith and S. S. Willenbrock, Phys. Rev. Lett. 79, 3825 (1997); M. Passera and A. Sirlin, Phys. Rev. D 58, 113010 (1998).

[18] B. A. Kniehl and A. Sirlin, Phys. Rev. D 77, 116012 (2008).

[19] B. A. Kniehl and A. Pilaftsis, Nucl. Phys. B474, 286 (1996).

[20] D. Espriu, J. Manzano, and P. Talavera, Phys. Rev. D 66, 076002 (2002).

[21] W. A. Bardeen, A. J. Buras, D. W. Duke, and T. Muta, Phys. Rev. D 18, 3998 (1978).

[22] C. G. Bollini and J. J. Giambiagi, Nuovo Cim. B 12, 20 (1972); G. 't Hooft and M. Veltman, Nucl. Phys. B44, 189 (1972); J. F. Ashmore, Lett. Nuovo Cim. 4, 289 (1972); Commun. Math. Phys. 29, 177 (1973).

[23] R. Hempfling and B. A. Kniehl, Phys. Rev. D 51, 1386 (1995).

[24] F. Jegerlehner, M. Yu. Kalmykov, and B. A. Kniehl, Phys. Lett. B 722, 123 (2013). 